\title{
Metastability and the Casimir Effect in Micromechanical Systems
}

\author{
E. Buks and M. L. Roukes \\ Condensed Matter Physics, California Institute of Technology, Pasadena, CA 91125
}

(October 6, 2018)

\begin{abstract}
Electrostatic and Casimir interactions limit the range of positional stability of electrostaticallyactuated or capacitively-coupled mechanical devices. We investigate this range experimentally for a generic system consisting of a doubly-clamped $\mathrm{Au}$ suspended beam, capacitively-coupled to an adjacent stationary electrode. The mechanical properties of the beam, both in the linear and nonlinear regimes, are monitored as the attractive forces are increased to the point of instability. There "pull-in" occurs, resulting in permanent adhesion between the electrodes. We investigate, experimentally and theoretically, the position-dependent lifetimes of the free state (existing prior to pull-in). We find that the data cannot be accounted for by simple theory; the discrepancy may be reflective of internal structural instabilities within the metal electrodes.
\end{abstract}

PACS numbers: 68.10.Cr, 68.35.Gy, 87.80.Mj

The technology of micro electro mechanical systems (MEMS) now routinely allows fabrication of miniature movable structures on-chip. This new branch of microelectronics enables the realization of a variety of miniature, fully integrated sensors and actuators, with a rapidly growing range of applications. One common building block within MEMS is the capacitively-tunable resonator (CTR), comprising a movable electrode that serves as a mechanical resonator, and an adjacent stationary electrode (see [1] and references therein). The capacitive coupling between the electrodes allows electrostatic control of both the mean position of the resonator and its resonance frequency. In addition to the electrostatic interaction, such electrodes are coupled via the Casimir effect [2], [3]. This quantum electrodynamical effect originates from the dependence of the ground state energy of the electromagnetic field upon boundary conditions and leads to an observable forces between macroscopic bodies. Due to its relatively short range, this force has only a small effect on the dynamics of macroscopic mechanical systems. However, the Casimir force can play a major role in modern MEMS where typical distances between neighboring surfaces can be on the submicron length scale [4]. These attractive electrostatic and Casimir forces give rise to a mechanical metastability that may cause stiction (for a review see [5], [6]). In the present case it is manifested as collapse (pull-in) of the movable resonator onto the nearby electrode, resulting in their permanent adhesion. This phenomena can be a principal cause of malfunctioning in MEMS. Moreover, it limits the range of stable operation of CTRs.

In the present work we study experimentally and theoretically the range of tunability and mechanical metastability in a CTR system made of a doubly clamped $\mathrm{Au}$ beam separated from an adjacent counterelectrode by a small vacuum gap. Once the beam is brought to contact with the electrode, permanent adhesion occurs. This indicates that the free state of this system is merely metastable, and that the state of contact (after pull-in) has lower energy due to the strongly attractive Casimir interaction. The potential barrier separating these two states determines the lifetime of the free state. The barrier can be reduced by introducing electrostatic attraction, namely by applying a DC voltage, $V_{d c}$ between the beam and the electrode. In our measurements we study the mechanical properties of the beam as $V_{d c}$ is gradually increased to the point of pull-in. Near this critical point all of the measured properties of the beam show strong dependence on $V_{d c}$. Using a simple model we calculate the shape of the potential barrier confining the metastable mechanical state, and then estimate the escape rate via both thermal excitation and quantum tunneling.

Fig. 1(a) is a micrograph displaying a side view of our device. The details of the fabrication process are given elsewhere [7]. The structure is designed to allow full characterization of the beam's properties to yield straightforward and unambiguous interpretation of our results. We use bulk (rather than surface) micromachining, which allows the substrate to be completely removed beneath the sample. This greatly simplifies the boundary conditions of the electromagnetic field in the vicinity of the sample. Moreover, we avoid using multilayered structures, since their internal stresses are generally important, and are difficult to model theoretically. The beam has length $l=200 \mu \mathrm{m}$, width $a=0.28 \mu \mathrm{m}$ and thickness $t=0.25 \mu \mathrm{m}$ (measured using an atomic force microscope). At its center, the stationary electrode has a $20 \mu \mathrm{m}$ long rectangular protrusion. This is separated from the beam by a vacuum gap of $g=1.28 \mu \mathrm{m}$.

All measurements are done at room temperature, insitu within a commercial scanning electron microscope (SEM). A voltage $V=V_{d c}+V_{a c} \cos (2 \pi \nu t)$ is applied between the beam and the electrode (see Fig. 1(b)). The static response of the beam is measured by operating the SEM in scanning mode, imaging the device, and using digital image processing to extract experimental data. The dynamical response is measured with a stationary electron beam focused at a point near the edge of the $\mathrm{Au}$ beam. To detect mechanical displacement we use a 
spectrum analyzer to monitor the output signal from the photomultiplier serving as a secondary electron detector. Note that this detection scheme is sensitive almost exclusively to motion in the plane of the sample.

To characterize the mechanical properties of the beam, namely stress and stiffness, we measure its resonance frequencies. For small stiffness they are given by:

$$
\nu_{n}=n \nu_{0}\left[1+2 \zeta+\left(4+n^{2} \pi^{2} / 2\right) \zeta^{2}\right],
$$

where $\zeta^{2}=E a^{3} t / 12 T l^{2}, \nu_{0}=\sqrt{T / \rho A} / 2 l$, with $E$ being Young's modulus, $T$ is the stress, and $\rho$ is the mass density [7], [8]. The dimensionless parameter $\zeta$ indicates the relative effect of stiffness compared to stress on the dynamics of the beam. Note that the terms that make the spectrum unequally spaced are of order $O\left(\zeta^{2}\right)$.

We excite the beam by applying $\mathrm{AC}$ voltage $\left(V_{d c}=0\right)$ and find three modes with frequencies $\nu_{1}=185.53 \mathrm{kHz}$, $\nu_{2}=372.4 \mathrm{kHz}$, and $\nu_{3}=563.8 \mathrm{kHz}$. Using Eq. (1) we find $\zeta=0.017 \pm 0.002$, thus stiffness has only a small effect on the dynamics of the beam. In what follows, we ignore altogether corrections due to stiffness. The stress is found from the measured resonance frequencies to be $T=7.4 \times 10^{-6} \mathrm{~N}$.

Next we gradually increase $V_{d c}$ and measure the mechanical properties of the beam until pull-in occurs. Figure 2(a) shows the normalized static deflection of the beam's center, $x_{1} / g$, as a function of $V_{d c}$. After completing the measurements at $V_{d c}=29 \mathrm{~V}$, pull-in occurred with this particular beam. Simultaneously applying the small AC excitation with the DC bias allows us to measure the frequency of the fundamental mode $\nu_{1}=\omega_{1} / 2 \pi$. Figure. 2(b) shows the ratio $-\Delta \omega / \omega_{0}=-\left(\omega_{1}-\omega_{0}\right) / \omega_{0}$, where $\omega_{0}$ is the angular frequency at $V_{d c}=0$. Both $x_{1}$ and $\omega_{1}$ show strong dependence on $V_{d c}$ near pull-in.

Further insight can be gained by studying the dynamics of the beam in the nonlinear regime. For oscillations with a large amplitude the harmonic approximation breaks down and effects due to higher order terms of the potential are observable. Figure 3 shows the measured response of a beam as a function of frequency around the fundamental resonance for a discrete range of ac drive $\left(V_{a c}\right)$ levels, at fixed DC bias, $V_{d c}=20 \mathrm{~V}$. As $V_{a c}$ is increased, the response becomes asymmetric, with a sharp drop occuring on the high frequency side. Moreover, a strong hysteresis is found for high $V_{a c}$ when the frequency is scanned back and forth across the resonance (see inset of Fig. 3). Theoretically, the response function in the nonlinear regime is given to lowest order by [9]:

$a^{2}\left[\left(\omega-\omega_{0}\left(1+\kappa a^{2}\right)\right)^{2}+\left(\omega_{0} / 2 Q\right)^{2}\right]=\left(\frac{f}{2 m \omega_{0}}\right)^{2}$,

where $f$ is the amplitude of the external force, $a$ is the amplitude of oscillations, $m$ is the mass, and $\kappa$ is a parameter describing the amplitude-dependent frequency shift due to nonlinearity.
From the shape of the resonance peaks in the nonlinear regime we extract the nonlinear frequency-shift parameter, $\kappa$, as a function of $V_{d c}$ (Fig. 2(c)). For relatively small $V_{d c}$ we find $\kappa>0$. For higher $V_{d c} \kappa$ becomes negative, and close to the point of pull-in $\kappa$ drops quite rapidly as a function of $V_{d c}$, indicating the strong nonlinearity of the potential in this regime.

We describe our system using a simple one dimensional model as proposed in Ref. 10 (see Fig. 4(a)). This model employs rather simplistic approximations and thus cannot be expected to provide accurate quantitative predictions - it is extremely useful, however, in providing physical insight and a qualitative description of the dynamics. A mass $m$ coupled to a string with a constant $k=m \omega_{0}^{2}$ represents the doubly-clamped beam. An adjacent surface at distance $g$ interacts with the mass electrostatically, as well as by the Casimir effect. For our device geometry it is appropriate to model these forces, $F$, as applied locally to the beam's center. In this case the displacement of the beam's center is $F l / 4 T$, and the effective spring constant is thus $k=4 T / l$.

The total potential energy is given by:

$$
U_{t}(x)=U_{E}(x)+U_{V}(g-x)+U_{C}(g-x) .
$$

The first term $U_{E}(s)=(1 / 2) k s^{2}$ is the beam's elastic potential. The second term is the electrostatic potential introduced by applying a DC voltage, $V$, between the beam and its counterelectrode. We estimate the capacitance $C$ between the beam and the electrode using a simple parallel plate approximation, thus $U_{V}(s)=-\epsilon_{0} A V^{2} / 2 s$, where $\epsilon_{0}$ is the vacuum permittivity and $A$ is the area. The third term is the Casimir potential. As in the electrostatic case, we employ a parallel plate approximation leading to $U_{C}(s)=\left(-\pi^{2} / 720\right)\left(\hbar c A / s^{3}\right)$, where $\hbar$ is the Planck's constant and $c$ is the velocity of light [3]. Using these expressions we rewrite $U_{t}$ in a dimensionless form:

$$
U_{t}=U_{E}(g)\left[\left(\frac{x}{g}\right)^{2}-\frac{\alpha}{1-x / g}-\frac{\beta}{1-(x / g)^{3}}\right],
$$

where $\alpha=-U_{V}(g) / U_{E}(g)=\epsilon_{0} A V^{2} / k g^{3}$ represents the relative importance of the electrostatic interaction (compared with the elastic term), and similarly $\beta=$ $-U_{C}(g) / U_{E}(g)=\left(\pi^{2} / 360\right)\left(\hbar c A / k g^{5}\right)$ for the ratio of Casimir interaction to the elastic term.

For relatively small values of $\alpha$ and $\beta$ (see figure 4(b)) the potential energy has a local minimum at $x=x_{1}$ associated with a metastable state. Figure 4(c) shows a typical example of $U_{t}$ in the metastable regime. The angular frequency of small oscillations around the local minimum is $\omega_{1}$. The barrier that separates the local minimum at $x=x_{1}$ from the global minimum at $x=$ $g$ has height $U_{b}$. Below, we calculate the parameters $\omega_{1}$ and $U_{b}$ for two special cases relevant to the present experiment. 
Consider first the case of $\alpha \ll 1$ and $\beta \ll 1$. In this case we find $x_{1} / g=(\alpha+3 \beta) / 2$ and $\omega_{1} / \omega_{0}=$ $(1-\alpha / 2-3 \beta)$. Since the escape rate for this case is very small, we do not estimate the associated $U_{b}$.

In the next case we consider the instability occurring with a strong electrostatic and a weak Casimir interaction. Note first that if $\beta=0$ then a local minimum and a potential barrier exist only for $\alpha<8 / 27$. At the critical point, $\alpha=8 / 27$, the local minimum and the local maximum points coincide at $x / g=1 / 3$. We therefore expand the potential near that point, assuming $\epsilon \equiv 8 / 27-\alpha \ll 1$ $(\epsilon>0)$ and also $\beta \ll 1$. To lowest order we find

$$
\begin{aligned}
x_{1} / g & =1 / 3-(1 / 8) \sqrt{32 \epsilon-216 \beta} \\
\omega_{1} / \omega_{0} & =(3 / 4)(32 \epsilon-216 \beta)^{1 / 4} \\
U_{b} / k g^{2} & =(3 / 512)(32 \epsilon-216 \beta)^{3 / 2}
\end{aligned}
$$

The critical point is thus shifted to $\alpha=8 / 27-27 \beta / 4$ due to the Casimir effect. The nonlinear frequency-shift parameter $\kappa$ is calculated from the coefficients of the Taylor expansion of the potential around the point $x=x_{1}$ [9]. To lowest order we find $\kappa g^{2} \propto(32 \epsilon-216 \beta)^{-3 / 4}$.

To compare the above results with theory we substitute the area, $A$, with an effective area, $A_{\text {eff }}=2.3 A$, chosen to ensure that the measured and calculated critical points coincide. This substitution serves to account, in part, for the crude approximations employed in the model. The solid lines in Fig. 2(a) and 2(b) show the theoretical results of Eq. (5a). Agreement with experiment for both $x_{1}$ and $\omega_{1}$ is moderately good despite the simplicity of the model. Note that our measurements within the nonlinear regime are strongly affected by nonlinearity in the detector's response. Given that this is not properly accounted for in our model, we do not attempt comparison with theory for the parameter $\kappa$.

We now turn our attention to the lifetime of the metastable mechanical state before pull-in. At finite temperatures, thermal excitation above the energy barrier can allow the system to "escape" from the freelymoving to the pulled-in state. The rate for this generic type of process was first found by Kramers 12, via the Fokker-Planck equation. The thermal escape rate for the case of small damping is

$$
\Gamma_{t}=\frac{\omega_{1}}{Q} \frac{U_{b}}{k_{B} T} \exp \left(-\frac{U_{b}}{k_{B} T}\right) .
$$

This result is valid when $U_{b} / k_{B} T \gg 1$ and $U_{b} / k_{B} T \gg$ $1 / Q$. Alternatively, at low temperatures the system escapes via quantum tunneling [11]. Caldeira and Leggett (CL) [13] 14 have explored what is termed macroscopic quantum tunneling (MQT) in systems with many degrees of freedom. In the case of small damping they find that the MQT rate is

$$
\Gamma_{Q}=\omega_{1} \xi_{1} \sqrt{\frac{U_{b}}{\hbar \omega_{1}}} \exp \left[-\frac{U_{b}}{\hbar \omega_{1}}\left(\xi_{2}+\xi_{3} / Q\right)\right] .
$$

Here $\xi_{1}, \xi_{2}$ and $\xi_{3}$ are positive constants of order unity. The rate for the case of no dissipation $(1 / Q=0)$ coincides with the conventional WKB result for tunneling within a noninteracting, single-particle picture. The CL model yields a description of the effect of dissipation upon MQT involving only a single parameter, $Q$, that subsumes all of the precise details of the interaction between the beam and the environment. Equation (7) explicitly demonstrates that the effect of dissipation is to suppress the tunneling rate.

In the present experiment the temperature is relatively high $\left(k_{B} T \gg \hbar \omega_{1}\right)$ therefore the rate of thermal escape is much larger compared to the rate of quantum tunneling. However, the opposite situation, namely $\Gamma_{Q} \gtrsim \Gamma_{t}$, may be realized with present-day technology. For high frequency mechanical oscillators (see for example [15]) the quantum limit, $k_{B} T \lesssim \hbar \omega_{1}$, is reachable at cryogenic temperatures. The model employed in the present paper may be useful in designing an experiment to study phenomena in this regime.

Comparison between the simple theory and experiment indicates significant disagreement. We extract the parameter $32 \epsilon-216 \beta$ from Eq. (5b) and the value $\omega_{1} / \omega_{0}=0.81$ found experimentally when the pull-in occurs. This parameter is substituted in Eq. (5c) to find $U_{b}$. This yields $U_{b} / k_{B} T \simeq 10^{7}$, a value which, when substitited into Eq. (6), yields a lifetime that is essentially infinite for all practical purposes. Experimentally, however, we observed pull-in after only a few minutes, in striking contradiction with this theoretical estimate. Similar devices, having $g=1.29,0.71$ and $0.65 \mu \mathrm{m}$, exhibited pull-in when $\omega_{1} / \omega_{0}=0.84,0.987$, and 0.995 respectively. In these cases, again, the simple theory is far from explaining what is experimentally observed. We note, in general, that any theory predicting $U_{b}=A k g^{2}\left(\omega_{1} / \omega_{0}\right)^{B}$ near the critical point, where both $A$ and $B$ are of order unity, will exhibit similar discrepancy. This is due to the exponential dependence in Eq. (6), in concert with the fact that $k g^{2} / k_{B} T \simeq 10^{7} \gg 1$.

Our results do not appear to be anomalous. We have analyzed data from previous experiments involving CTR devices made from stress-free silicon [1], in which pullin was also observed upon application of a DC voltage between electrodes. Calculating the expected lifetime for the experimental conditions of Ref. [1] using Eq. (6), we find lifetimes that are in similarly gross contradiction with experimental observations. In principle, slow processes of stress relaxation in both cases may be the underlying mechanism inducing early pull-in [16]. Further investigations are warranted. In our work we have taken care to rule out spurious effects from environmental electrical noise sources that could, in principle, drive the system out of the metastable state. Measured electrical noise was far smaller than the steady bias voltages applied. Also, "early" pull-in occurred even when the electron beam was blanked, ruling out the possibility that 
some form of parasitic local charging may be operative.

The present work shows that MEMS can provide an ideal approach to studies of mechanical metastability. Further experimental and theoretical work will elucidate the underlying mechanisms governing the instability of these systems. Such work is important not only from a theoretical point of view, but also from the standpoint of practical device engineering. Moreover, further enhancement of experimental techniques will enable new studies of macroscopic quantum tunneling in mechanical systems.

The authors are grateful to K. Schwab for his assistance in sample fabrication. This research was supported by DARPA MTO/MEMS under grant DABT6398-1-0012. E.B. gratefully acknowledges suppport from a Rothschild Fellowship and the R.A. Millikan Postdoctoral Fellowship at Caltech.

[1] S. G. Adams, F. M. Bertsch, K. A. Shaw, P. G. Hartwell, F. C. Moon and N. C. MacDonald, J. Micromech. Microeng. 8, 15 (1988).

[2] H. B. G. Casimir, Proc. Kon. Ned. Akad. Wetenschap 51, 793 (1948).

[3] S. K. Lamoreaux, Am. J. Phys. 67, 850 (1999).

[4] F. Michael Serry, Dirk Walliser and Jordan Maclay, J. Appl. Phys. 84, 2501 (1998).

[5] Niels Tas, Tonny Sonnenberg, Henri Jansen, Rob Legtenberg and Miko Elwenspoek, J. Micromech. Microeng. 6, 385 (1996).

[6] Roya Maboudian and Roger. T. Howe, J. Vac. Sci. Technol. B15, 1 (1997).

[7] E. Buks and M. L. Roukes, cond-mat/0008051.

[8] L. D. Landau and E. M. Lifshitz, Theory of Elasticity (Pergamon 1986).

[9] L. D. Landau and E. M. Lifshitz, Mechanics (Pergamon 1976).

[10] F. Michael Serry, Dirk Walliser and G. Jordan Maclay, J. Micro. Elec. Mech. 4, 193 (1995).

[11] A. N. Cleland and M. L. Roukes, unpublished (1996); A. N. Cleland and M. L. Roukes, in 24th International Conference on the Physics of Semiconductors, Ed. David Gershoni (World Scientific, 1999).

[12] H. A. Kramers, Physica 7, 284 (1940).

[13] A. O. Caldeira and A. J. Leggett, Phys. Rev. Lett. 46, 211 (1981).

[14] A. O. Caldeira and A. J. Leggett, Ann. Phys. 149, 374 (1983).

[15] Vu Thien Binh, N. Garcia, and A. L. Levanuky, Surf. Sci. Lett. 301, L224 (1994).

[16] Hoo-Jeong Lee, Guido Cornella and John C. Bravman, App. Phys. Lett. 76, 3415 (2000).
FIG. 1. (a) Side view micrograph of the device. (b) External force is applied by a voltage bias and motion is detected by focusing the e-beam on a point on the edge of the structure and measuring the secondary reflected electrons.

FIG. 2. (a) Displacement of the center of the beam $x_{1}$ divided by $g$. (b) Frequency shift $\Delta \omega$ divided by the unperturbed frequency $\omega_{0}$. (c) Nonlinear parameter $\kappa g^{2}$.

FIG. 3. The mechanical response as a function of frequency around the fundamental resonance with $V_{d c}=20 \mathrm{~V}$ and $V_{a c}=25,50,75, \ldots, 225 \mathrm{mV}$. The inset shows the hysteresis loop for $V_{a c}=225 \mathrm{mV}$ when the frequency is scanned forth and back across the resonance.

FIG. 4. (a) A one dimensional model describing our system. (b) the metastable regime in the $\alpha \beta$ plane. (c) The potential energy in the metastable regime has a barrier $U_{b}$ separating the metastable state at $x=x_{1}$ and the contact state at $x=g$. 